\title{
Phase dependence mitigation for autocorrelation-based frequency estimation
}

\author{
Mustafa A. Altınkaya ${ }^{\mathrm{a}, *}$, Emin Anarım ${ }^{\mathrm{b}}$, Bülent Sankur ${ }^{\mathrm{b}}$ \\ a Department of Electrical and Electronics Engineering, Faculty of Engineering, Izmir Institute of Technology, İzmir, Turkey \\ ${ }^{\mathrm{b}}$ Department of Electrical and Electronics Engineering, Faculty of Engineering, Boğaziçi University, İstanbul, Turkey
}

Available online 22 February 2007

\begin{abstract}
The sinusoidal frequency estimation from short data records based on Toeplitz autocorrelation (AC) matrix estimates suffer from the dependence on the initial phases of the sinusoid(s). This effect becomes prominent when the impact of additive noise vanishes, that is at high signal-to-noise ratios (SNR). Based on both analytic derivation of the AC lag terms and simulation experiments we show that data windowing can mitigate the limitations caused by the phase dependence. Thus with proper windowing, the variance of the frequency estimate is no more eclipsed by phase dependence, but it continues to decrease linearly with increasing SNR. The study covers both the cases of a single sinusoid and two sinusoids closely spaced in the frequency with the Pisarenko frequency estimator, MUSIC and principal component autoregressive frequency estimators. The trade-offs between the spectral broadening and the achieved minimum variance level due to the data window are analyzed in detail.
\end{abstract}

(c) 2007 Elsevier Inc. All rights reserved.

Keywords: Frequency estimation; Data windowing; Subspace methods; Autocorrelation method; Phase dependence

\section{Introduction}

Model-based frequency estimation methods are used extensively due to their computational advantages and high resolution property. Especially, for short data records model-based spectral estimation techniques show superior performance under adequate signal-to-noise ratio (SNR) conditions.

Although the methods that specifically make use of the Toeplitz autocorrelation (AC) matrix, that is the AC method of linear prediction, exhibit good performance at low SNRs, they suffer from the dependence on the initial phase(s) of the sinusoid(s). This phase dependence reveals itself at high SNR values as a phenomenon similar to noise floor effect. In other words, even though the influence of the additive noise has been reduced to a negligible level, the accuracy of the frequency estimate does not improve proportional to the ever increasing SNR.

An approach to obviate this handicap is to use the non-Toeplitz versions of the AC matrix, that is the covariance method of linear prediction [1]. These non-Toeplitz spectrum/frequency estimation schemes show superior perfor-

\footnotetext{
A compact version of this paper was presented in The 2005 European Signal Processing Conference (EUSIPCO-2005), Antalya, Turkey, September 4-8, 2005.

* Corresponding author. Fax: +90 2327506505.

E-mail addresses: mustafaaltinkaya@ iyte.edu.tr (M.A. Altınkaya), anarim@boun.edu.tr (E. Anarım), bulent.sankur@boun.edu.tr (B. Sankur).
} 
mance vis-a-vis their Toeplitz companions under phase uncertainty conditions. However, computing the eigenvalues and eigenvectors of non-Toeplitz, that is, symmetric but arbitrary real matrices, is of $O\left(M^{3}\right)$ complexity [2] while the complexity of Toeplitz matrices remains at order $O\left(M^{2}\right)$ [3], where the notation $O(\cdot)$ denotes the order of required complex multiplications and $M$ is the size of the $\mathrm{AC}$ matrices. This complexity differential motivates us for subspace-based frequency estimators utilizing Toeplitz AC matrix estimates.

In this work, we investigate the performance trade-offs of data windowing to reduce phase dependence, in other words to promote the performance of AC methods up to the level of covariance methods, and the concomitant loss of resolution. The highlights of our work lists as follows:

- A data windowing scheme is proposed that decreases the phase dependence of the AC lag estimates, which in turn were responsible for the variance lower bound in the "Toeplitz" frequency estimation. The trade-offs between power loss, resolution and variance reduction of the windows are demonstrated for the single and double tone cases.

- The statistics of AC lag estimates with and without data windowing in the case of a single real sinusoid are derived. This probability density function (pdf) is found to be the convolution of a Gaussian pdf due to additive white Gaussian noise (AWGN) and a double-peaked pdf due to the phase dependence.

- The histogram of the Pisarenko frequency estimator (PISFE) for the single tone case is shown to have a doublepeaked form similar to the pdfs of AC lags in high SNR conditions. Windowing results in a similar mitigation effect for PISFE as well. Consequently, the derived pdf of PISFE, which is based on the Gaussianity assumption of the AC lags after data windowing, perfectly matches the simulated histogram.

The paper is organized as follows: In Section 2, the sinusoidal frequency estimation problem and the selected frequency estimators, namely, PISFE, multiple signal characterization (or classification) (MUSIC) and the principal component autoregressive (PC-AR) frequency estimators are briefly visited. In Section 3, the need for data windowing is justified and the statistics of the AC lag estimates with and without data windowing are derived. The analytical expressions for AC lags are compared with simulation results for validation. In Section 4, the pdfs of PISFE with and without data windowing are derived and the related performance improvement is discussed. Section 5 covers the simulation results with both single and two sinusoids to demonstrate the trade-offs in selecting a suitable data window. Finally, conclusions are given in Section 6. Detailed derivations of the pdf of the phase-dependent component of AC lags, the pdf of AC lags with data windowing and the pdf of PISFE are given in the appendices.

\section{Frequency estimation problem and utilized model-based estimators}

The signal model under consideration consists of multiple real sinusoids observed in AWGN, i.e.,

$$
x_{k}=s_{k}+n_{k}=\sum_{i=1}^{K} \sqrt{2 A_{i}} \cos \left[\omega_{i} k T+\phi_{i}\right]+n_{k}, \quad k=1,2, \ldots, N,
$$

where $A_{i}, \phi_{i}$, and $\omega_{i}$ are the non-random amplitude, the random phase angle uniformly distributed on $(-\pi, \pi)$ and the angular tone frequency of the $i$ th real sinusoid, respectively, and $T$ is the sampling period, $\left\{n_{k}\right\}$ is a real white Gaussian noise sample sequence with zero mean and power $\sigma_{n}^{2}$ and $N$ is the number of data samples. We are interested only in the angular frequency parameter. The other parameters are considered as nuisance factors. We assume without loss of generality that the number of sinusoids is either known or can be estimated from the data. We also drop the index of the sinusoidal parameters for the single sinusoid case and we will assume $T=1$ throughout the paper in order to simplify the notation.

In this work we assess three different frequency estimators, namely, PISFE, MUSIC, and PC-AR frequency estimators. PISFE is an inherently Toeplitz sample AC matrix-based frequency estimator, generally used to estimate the frequency of single sinusoids [4,5]. Its mathematical tractability enables us to derive its pdf (see Section 4), which in turn makes it possible to obtain analytical quality expressions for the frequency estimates.

The single-dimensional noise-subspace of PISFE is responsible for the rather poor performance of this estimator. Therefore, we look at two alternative frequency estimators, MUSIC and PC-AR, which use higher dimensional noisesubspaces. MUSIC is the widely used generalization of Pisarenko harmonic decomposition concept and it reduces to 
PISFE when the dimension of noise-subspace is unity [6]. PC-AR frequency estimator [1] is based on signal-subspace quantities in contrast to MUSIC and PISFE. We use MUSIC and PC-AR frequency estimators to show that the advantages of data windowing, proven analytically for PISFE, generalizes to more powerful frequency estimators. In order to obtain the MUSIC and PC-AR frequency estimators, the Toeplitz sample AC matrix must first be constructed as

$$
\mathbf{R}_{M}=\left[\begin{array}{cccc}
r(0) & r(-1) & \cdots & r(-M+1) \\
r(1) & r(0) & \cdots & r(-M+2) \\
\cdots & \cdots & \cdots & \cdots \\
\cdots & \cdots & \cdots & \cdots \\
r(M-1) & r(M-2) & \cdots & r(0)
\end{array}\right],
$$

where $r(k)$ denotes the $k$ th autocorrelation coefficient of the input samples and is estimated as

$$
r(k)=\frac{1}{N-k} \sum_{i=1}^{N-k} x_{i} x_{i+k} .
$$

\section{The description of the phase dependence}

Consider the sample AC coefficients, given via the AC method as in (3). For a single sinusoid at angular frequency $\omega$, the AC lag estimates are given by (assume $A=1$ without loss of generality)

$$
\begin{aligned}
r_{x}(l)= & \frac{1}{N-l} \sum_{k=1}^{N-l}\left\{\cos (\omega l)+\cos (\omega(2 k+l)+2 \phi)+\left(\sqrt{2} \cos (\omega k+\phi) n_{k+l}\right)\right. \\
& \left.+\left(\sqrt{2} \cos (\omega(k+l)+\phi) n_{k}\right)+n_{k} n_{k+l}\right\} .
\end{aligned}
$$

The large sample statistics of $r_{x}(l)$ was derived in $[7,8]$ which were shown to be Gaussian distributed with mean and variance

$$
\mu_{x}(l)=\cos (\omega l)
$$

and

$$
\sigma_{x}^{2}(l)=\frac{\sigma_{n}^{4}}{N}\left(1+\frac{4}{\sigma_{n}^{2}} \cos ^{2}(\omega l)\right)
$$

respectively, for $l=0, \ldots, N-1$. For small sample sizes let us express $r_{x}(l)$ as

$$
r_{x}(l)=r_{x, h}(l)+r_{x, g}(l),
$$

where

$$
r_{x, h}(l)=\frac{1}{N-l} \sum_{k=1}^{N-l} \cos (\omega(2 k+l)+2 \phi),
$$

and where $r_{x, g}(l)$ corresponds to the sum of all the remaining terms in $r_{x}(l)$, that is the deterministic component $\cos (\omega l)$ and the last three components which contain AWGN terms. In this study, we neglect the dependence of $r_{x, h}(l)$ and $r_{x, g}(l)$ and derive the pdf of $r_{x}(l)$ under the assumption that they are statistically independent. Indeed the sample crosscorrelation coefficients of $r_{x, h}(1)$ and $r_{x, g}(1)$ are found to be $-0.0022,-0.00075$, and -0.00058 at 0 , 20, and $40 \mathrm{~dB}$ SNRs, respectively. These negligible values justify our independence approximation. Furthermore we assume that the additive noise components dominate the $r_{x, g}(l)$ term and that the effect of phase is secondary. The pdf of the sum of two independent random variables in (6) is obtained as the convolution of the pdfs of the individual component variables. The pdf of $r_{x, h}(l)$ is derived in Appendix A as

$$
f_{R_{x, h}}^{(l)}\left(r_{x, h}\right)=\frac{1}{\pi d_{x}(l) \sqrt{1-\left(r_{x, h}(l) / d_{x}(l)\right)^{2}}} \text { for }-d_{x}(l)<r_{x, h}(l)<d_{x}(l),
$$

where

$$
d_{x}(l)=\frac{1}{N-l}\left|\sum_{k=1}^{N-l} \exp \{j(\omega(2 k+l))\}\right| .
$$


The term, $r_{x, h}(l)$, which takes a convolutive role in the pdf of the Toeplitz AC lag estimates given in (7), is a direct outcome of the phase dependence. This pdf has the form of the derivative of the $\arcsin \omega$ scaled with the factor $d_{x}(l)$. Notice first, that $d_{x}(l)$ determines the support of the pdf in (7), and second, that the support will tend to zero slowly due to the factor $1 /(N-l)$. As for the second term in $(6), r_{x, g}(l)$, we assume it to have a Gaussian distribution, valid for large sample sizes, with mean $\mu_{x}(l)$ and variance $\sigma_{x}^{2}(l)$. So, the pdf of $r_{x}(l)$ is found as the convolution

$$
f_{R_{x}}^{(l)}\left(r_{x}\right)=\int_{-d_{x}(l)}^{d_{x}(l)} \frac{\exp \left\{-\frac{1}{2} \frac{\left(r_{x}(l)-\tau-\mu_{x}(l)\right)^{2}}{\sigma_{x}^{2}(l)}\right\}}{\pi d_{x}(l) \sqrt{1-\left(\tau / d_{x}(l)\right)^{2}} \sqrt{2 \pi \sigma_{x}^{2}(l)}} d \tau .
$$

In Figs. 1 and 2 we plotted the pdf of $r_{x}(1)$ together with the simulations histogram with 100,000 independent runs of a single sinusoid in AWGN where $\omega=\pi / 4 \mathrm{rad}$ and $N=50$. In Fig. 1, we have SNR $=5 \mathrm{~dB}$ whereas it is $30 \mathrm{~dB}$ in Fig. 2. We observe close matching of the histogram and the Gaussian pdf (mean $\mu_{x}(1)$ and variance $\left.\sigma_{x}^{2}(1)\right)$ in Fig. 1, even though this pdf was derived using the central limit theorem (CLT), which is actually valid for large sample sizes [7,8]. For the $5 \mathrm{~dB}$ SNR value, the pdf computed using (9) perfectly matches the Gaussian pdf. For some higher SNR value, say $30 \mathrm{~dB}$, the pdf of $r_{x}(1)$ as depicted in Fig. 2 no longer resembles to a Gaussian form. In fact, its shape is



Fig. 1. Pdf of $r_{x}(1)$ : low SNR case, solid line: analytic computation, histogram lines: histogram obtained by simulation $(\omega=\pi / 4 \mathrm{rad}, N=50$, $\mathrm{SNR}=5 \mathrm{~dB}, 100,000$ noise realizations).

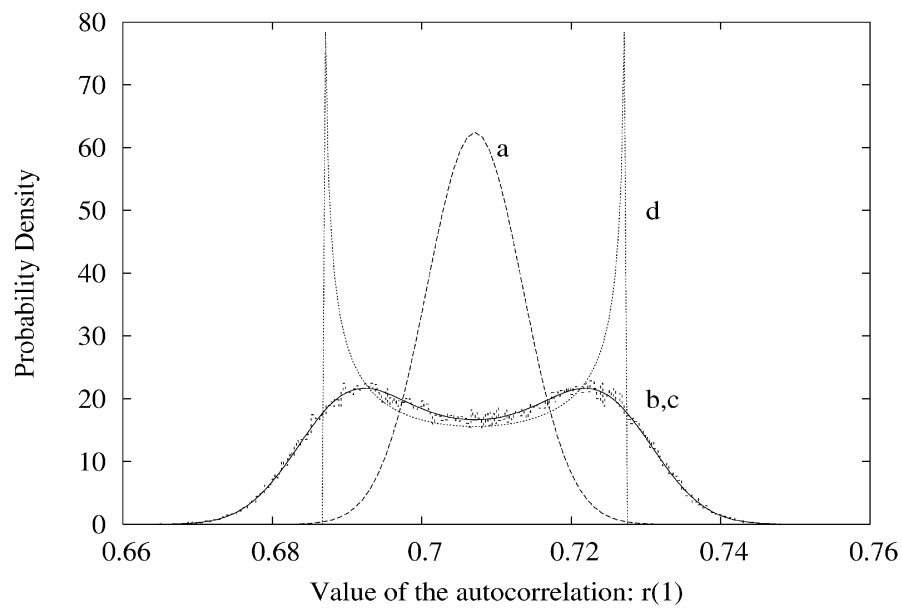

Fig. 2. Pdf of $r_{x}$ (1): high SNR case, a: pdf of $r_{x, g}$ (1) (only Gaussian component, analytic computation), b: pdf of $r_{x}$ (1) (analytic computation), c: histogram obtained by simulation, d: pdf of $\left(r_{x, h}(1)+\cos (\omega)\right)$ (shifted phase-dependent component, analytic computation) $(\omega=\pi / 4$ rad, $N=50, \mathrm{SNR}=30 \mathrm{~dB}, 100,000$ noise realizations). 
governed by the pdf of the phase-dependent term $r_{x, h}(1)$ whose support is independent from SNR level. This behavior makes it impossible for the variance of the AC lag estimates to tend to zero as the SNR increases unboundedly. The asymptotic lower bound in this case is given by the variance of $r_{x, h}(l)$. This behavior will consequently cause an error floor on the variance of the frequency estimates. The pdf of $r_{x}(1)$ computed by (9) and the simulation histogram again match closely as shown by the curves "b" and "c" in Fig. 2. In this figure the pdfs of the individual Gaussian and phasedependent components are also plotted, which use, respectively, the Gaussian pdf assumption and expression (7) with $l=1$.

\subsection{Data windowing to alleviate the effects of phase dependence}

We suggest data windowing (or tapering) to alleviate the effect of phase dependence on the frequency estimators based on Toeplitz sample AC matrix. We point out that the phase dependence becomes observable only at large SNRs and that it should be considered as a leakage phenomenon in the classical sense, that is, the smearing of power spectrum due to discontinuities in the periodic boundaries [9]. For a pure sinusoid, one can observe that the distribution of leakage in the power spectrum is a function of the tone phase. Based on the Wiener-Khinchine relationship between the AC lags and the power spectrum, the frequency estimation using AC lags is equivalent to DFT-based methods. Thus one can argue that, data windowing which is the standard leakage control technique in DFT-based spectra, should be effective in mitigating the leakage disturbance due to phase dependence in the AC lags. On the other hand, AC lags-based frequency estimation shares computational advantage and also partly the high resolution property of model-based estimators when compared to DFT analysis-based methods as it will be shown in the sequel.

When the data terms in (1) are multiplied by the window coefficients $\left\{w_{k}, k=1, \ldots, N\right\}$, we denote the windowed process samples as $\left\{y_{k}, k=1, \ldots, N\right\}$, so that the AC lag terms $\left\{r_{x}(l), r_{x, h}(l), r_{x, g}(l)\right\}$ are now denoted as $\left\{r_{y}(l), r_{y, h}(l), r_{y, g}(l)\right\}$. Obviously, for the rectangular window one has $\left\{y_{k} \equiv x_{k}, k=1, \ldots, N\right\}$. The statistics of the $\mathrm{AC}$ lags for windowed data are derived in Appendix B. The mitigation of the phase-dependence reveals itself in the shape of the AC lag pdfs. In fact the windowing causes simply the shrinking of the support of the phase-dependent variable $r_{x, h}(l)$. After windowing, the support of the phase-dependent term, $r_{y, h}(l)$, is given by

$$
d_{y}(l)=\frac{1}{N-l}\left|\sum_{k=1}^{N-l} w_{k} w_{k+l} \exp \{j(\omega(2 k+l))\}\right|,
$$

where $j=\sqrt{-1}$. For a rectangular window an analytic closed form expression can be found easily. For a general window, though analytical expression cannot be obtained, the multiplicative windowing factors in (10) become bounded by

$$
0 \leqslant w_{k} w_{k+l} \leqslant 1
$$

Finally, this term becomes approximately equal to 1 in the middle of each window and it tends to zero at the boundaries of the taper. For all reasonable window functions, the condition in (11) will hold and $d_{y}(l)$ will get smaller the smoother the data window is. The computed values of $\left\{d_{y}(l), l=1,2,3\right\}$ for $N=50$ and $\omega=0.5$ rad are listed in Table 1, which justify our claim.

The AC lags of the windowed data will still have a Gaussian part $r_{y, g}(l)$ with mean and variance given by (B.2) and (B.6), respectively. In conclusion, with data windowing, the pdfs of both the $r_{x, h}(l)$ and $r_{x, g}(l)$ terms maintain their shape, but with modified parameters $d_{y}(l), \mu_{y, g}(l)$, and $\sigma_{y, g}^{2}(l)$.

Restating the phase dependence problem in the frequency estimation, the pdf of $r_{x}(l)$ appears as a twin-peaked function, especially at high SNR and small sample-size-values. Recall that the separation of the peaks in (7) is given by $d_{x}(l)$ value (a direct evidence of phase dependence), and the two Gaussian humps have widths proportional to the

Table 1

$\left\{d_{y}(i), i=1,2,3\right\}$ for the data windows $(N=50, \omega=0.5 \mathrm{rad})$

\begin{tabular}{lllll}
\hline Window name & Rectangular & Hamming & Raised cosine & Blackman-Harris \\
\hline$d_{y}(1)$ & 0.025173 & 0.00016380 & $2.2832 \mathrm{E}-06$ & $6.3767 \mathrm{E}-08$ \\
$d_{y}(2)$ & 0.039352 & 0.00028108 & $2.9835 \mathrm{E}-06$ & $6.6067 \mathrm{E}-08$ \\
$d_{y}(3)$ & 0.044294 & 0.00035629 & $5.6746 \mathrm{E}-06$ & $6.9180 \mathrm{E}-08$ \\
\hline
\end{tabular}


Table 2

Some common data windows

\begin{tabular}{ll}
\hline Window name & Discrete-time function $w_{k}(k=0, \ldots, N-1)$ \\
\hline Rectangular (uniform) & 1 \\
Hamming & $0.54+0.46 \cos \left(\frac{2 \pi}{N} k\right)$ \\
Raised cosine (Hann, with $\alpha=2)$ & $0.5+0.5 \cos \left(\frac{2 \pi}{N} k\right)$ \\
Blackman-Harris $^{\mathrm{a}}$ & $\frac{10}{32}+\frac{15}{32} \cos \left(\frac{2 \pi}{N} k\right)+\frac{6}{32} \cos \left(\frac{4 \pi}{N} k\right)+\frac{1}{32} \cos \left(\frac{6 \pi}{N} k\right)$ \\
\hline
\end{tabular}

a The given Blackman-Harris type window is designed to achieve continuous first fifth order derivatives at the boundaries [11].

additive noise variance. There are three ways to merge the twin peaks into one that remove the evidence of phase dependence. The first method is to increase the number of samples such that the valley width between peaks shrinks with rate $1 /(N-l)$. In the second method, merging can be attained with excess Gaussian smearing (dominance of the Gaussian term in the convolution), as would happen at low SNRs, e.g., in Fig. 1. Finally the third method, as proposed in this paper (curves "b" and "c" in Fig. 2), is windowing, where $\left\{d_{y}(l), l=1, \ldots, M\right\}$, the supports of $f_{R_{y, h}}^{(l)}\left(r_{y, h}\right)$, shrink and finally the small gap is filled by the Gaussian smear (convolution). Thus, improvement in the phase dependence is obtained without sacrificing SNR or being obliged to take more data, however, one sacrifices resolution.

The window types used in this work are given in Table 2.

\section{The pdf of PISFE}

Since PISFE allows analytical derivation of its pdf, we used it as a test case to illustrate the mitigation of the phase dependence via windowing. For a single sinusoid case, PISFE is obtained in terms of the AC coefficients as follows [10]:

$$
\hat{\omega}_{\text {PISFE }}=\arccos (\psi),
$$

where

$$
\psi=\frac{r(2)+\sqrt{r^{2}(2)+8 r^{2}(1)}}{4 r(1)} .
$$

The pdf of PISFE is obtained from the pdf of the intermediate random variable $\psi$ using the transformation

$$
f\left(\hat{\omega}_{\mathrm{PISFE}}\right)=\sqrt{1-\cos ^{2}\left(\hat{\omega}_{\mathrm{PISFE}}\right)} f_{\Psi}\left(\cos \left(\hat{\omega}_{\mathrm{PISFE}}\right)\right) .
$$

The pdf of $\psi$ is derived in Appendix C. The derivations are based on the CLT so the pdf expression is expected to be valid only for large $N$ (e.g., $N \geqslant 50$ ). The pdf is

$$
f_{\Psi}(\psi)=\frac{4 K_{1} e^{-A_{4} / 2}}{A_{2}(\psi)}\left[\frac{B_{1}(\psi) u(\psi)}{\left(1+A_{1}(\psi)\right)}+\frac{B_{2}(\psi) u(-\psi)}{\left(1-A_{1}(\psi)\right)}\right],
$$

where $u(\cdot)$ represents the unit step function. The remaining definitions are given as

$$
\begin{aligned}
& K_{1}=\frac{1}{2 \pi \sigma_{1} \sigma_{2} \sqrt{1-\rho^{2}}}, \\
& A_{0}(\psi)=\left(2 \psi^{2}-1\right) / \psi, \\
& A_{1}(\psi)=A_{0}(\psi) / \sqrt{A_{0}^{2}(\psi)+8}, \\
& A_{2}(\psi)=\frac{1}{\left(1-\rho^{2}\right)}\left[\left(\frac{A_{0}(\psi)}{\sigma_{2}}\right)^{2}+\frac{1}{\sigma_{1}^{2}}-\frac{2 \rho A_{0}(\psi)}{\sigma_{1} \sigma_{2}}\right], \\
& A_{3}(\psi)=\frac{2}{\left(1-\rho^{2}\right)}\left[\frac{\rho}{\sigma_{1} \sigma_{2}}\left(\mu(2)+A_{0}(\psi) \mu(1)\right)-\frac{\mu(1)}{\sigma_{1}^{2}}-\frac{A_{0}(\psi) \mu(2)}{\sigma_{2}^{2}}\right],
\end{aligned}
$$




$$
\begin{aligned}
& A_{4}=\frac{1}{\left(1-\rho^{2}\right)}\left[\left(\frac{\mu(1)}{\sigma_{1}}\right)^{2}+\left(\frac{\mu(2)}{\sigma_{2}}\right)^{2}-\frac{2 \rho \mu(1) \mu(2)}{\sigma_{1} \sigma_{2}}\right], \\
& B_{1}(\psi)=\left[1+\frac{A_{3}(\psi)}{4} \sqrt{\frac{2 \pi}{A_{2}(\psi)}} \exp \frac{A_{3}^{2}(\psi)}{8 A_{2}(\psi)}\left(\operatorname{erf}\left(\frac{A_{3}(\psi)}{\sqrt{8 A_{2}(\psi)}}\right)-1\right)\right], \\
& B_{2}(\psi)=\left[1+\frac{A_{3}(\psi)}{4} \sqrt{\frac{2 \pi}{A_{2}(\psi)}} \exp \frac{A_{3}^{2}(\psi)}{8 A_{2}(\psi)}\left(\operatorname{erf}\left(\frac{A_{3}(\psi)}{\sqrt{8 A_{2}(\psi)}}\right)+1\right)\right]
\end{aligned}
$$

with $\{\mu(i), i=1,2\}$ and $\left\{\sigma_{i}^{2}=\sigma^{2}(i), i=1,2\right\}$ representing the means and variances of the first two AC lags given by (4) and (5), respectively, and

$$
\rho=\frac{\operatorname{cov}(r(1), r(2))}{\sigma_{1} \sigma_{2}}
$$

is their correlation coefficient, where

$$
\operatorname{cov}(r(1), r(2))=\left(\frac{4 A \sigma_{n}^{2}}{N}\right) \cos (\omega) \cos (2 \omega)
$$

is their cross-covariance. Finally, erf(.) denotes the error function.

We performed simulations with and without data windowing and also calculated analytically the corresponding pdf using (13) and (14). In the calculation of the pdf of PISFE, the pdf of the AC lags are modeled by the Gaussian densities with means and covariances defined by (4), (5), and (15) for the non-windowed data and by (B.2), (B.6), and (B.7) for the windowed data, respectively. In other words, the phase dependence was intentionally ignored in contrast to the simulation results. The normalized angular frequency $\omega=\pi / 4 \mathrm{rad}$, the sample size $N=100$ and the number of the independent runs in the simulation is 100,000. The results in Fig. 3 depict how, without data windowing, the phase-dependent effect emerges, even at the not very high SNR of $20 \mathrm{~dB}$. The mismatch between the analytical pdf and the simulation histograms is due to neglecting the phase-dependence effect. On the other hand, when a data window is utilized, the analytical pdf and the histogram match perfectly. When the computed pdfs with and without data windowing are compared, one can see a performance degradation introduced by the data window as the width of the pdf is increased. However, one should consider that the computed pdf with no data window is hypothetical since the Gaussianity assumption on the pdfs of the AC lag estimates fails, which in turn makes the pdf expression of PISFE based on this assumption invalid.

We used arbitrarily a Blackman-Harris (BH) type data window [9,11] defined in Table 2 which eliminated the splitting of the pdf.

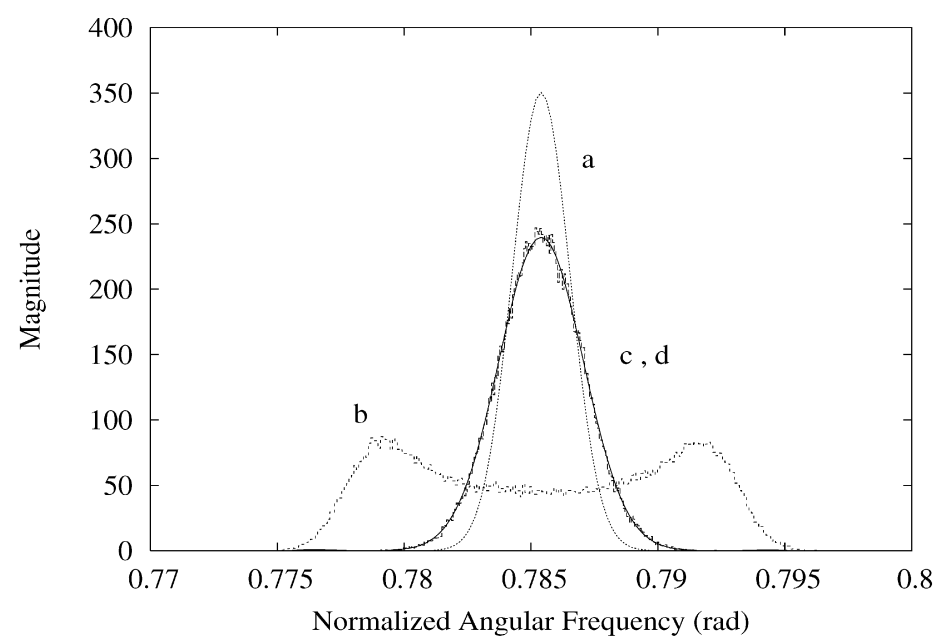

Fig. 3. Pdf of PISFE with and without data windowing versus normalized angular frequency, a: analytic computation for non-windowed data, b: histogram for non-windowed data simulation, $\mathrm{c}$ : analytic computation for windowed data, $\mathrm{d}$ : histogram for windowed data simulation $(\omega=\pi / 4$ rad, $N=100, \mathrm{SNR}=20 \mathrm{~dB}, \mathrm{BH}$ data window). 


\section{Choice of the data window}

Two important design parameters in spectrum estimation are the zero-bias and small-variance properties of the windows. Since the known bias introduced by windowing from the AC lags is removed, we can concentrate on the variance aspects of windowing for frequency estimation. We recall that the impact of bias with respect to variance to the mean square frequency error is much lower, anyway, for the estimators considered. The basic trade-offs of data windowing are as follows: For the single sinusoids, the trade-off is between the decrease in the effective SNR and the minimum required variance level of the estimate. For multiple sinusoids, resolution capability becomes another design parameter.

Windows like the $\mathrm{BH}$ window in Table 2 have very smooth endpoint transitions but in turn they may considerably decrease the signal power and the resolving capability of the frequency estimator. On the other hand, the rectangular window (refer to Table 2), i.e., no data window at all, does not cause any power loss but possesses a much higher variance lower bound. One should then choose a window compromising these two contradictory design objectives. Assuming that $r_{y, h}(l)$ and $r_{y, g}(l)$ are independent, the variance of their sum is lower bounded by the sum of variances of each component. So, in Table 3, we calculate analytically the variances of the phase-dependent components $\left\{r_{y, h}(l), l=\right.$ $1,2,3\}$ for four window varieties and sample sizes $N$ for $\omega=0.5 \mathrm{rad}$. The first column, in fact, represents the absence of windowing. For each sample size and windowing function, the column of three numbers top to bottom correspond to $r_{y, h}(1), r_{y, h}(2)$, and $r_{y, h}(3)$. The variances are calculated using (7) with support limits calculated using (10).

Table 3 depicts that, in general, for every window, the variance of the phase dependent AC lag component decreases with increasing sample size and, for a fixed sample size, the variance drops as a smoother window is used. The computed values of the support limits $\left\{d_{y}(l), l=1,2,3\right\}$ for $N=50$ and $\omega=0.5 \mathrm{rad}$, listed in Table 1 , are in accordance with the variance figures of the second row in Table 3. The variance figures smaller than $-100 \mathrm{~dB}$ are given in bold font to discriminate the cases where we assume that the residual phase dependence is negligible. It is important that without data windowing, i.e., with a rectangular data window, the variance figures are as high as approximately $-60 \mathrm{~dB}$ even for a very large sample size of $N=1000$. This means that phase dependence problem cannot be solved with increased sample size.

In Table 3, we observe some variance figures which do not obey the general trends mentioned above and should be explained by different mechanisms. In the first category of these exceptional results, when $(N-l)$ is nearly a multiple of the period, $\pi / \omega$, the phase dependence tends to vanish. This effect is observed in $r_{y, h}(1)$ in the rectangular window case when $N=20$ and 1000 and in the Hamming window case when $N=1000$. Notice that in the rectangular window case the variance of $r_{y, h}(1)$ when $N=20$ and when $N=200$ are nearly equal. The variances of $r_{y, h}(1), r_{y, h}(2)$, and

Table 3

$-10 \log$ (variance) figures for the phase-dependent component of AC lags $r_{x, h}(1)$ (top), $r_{x, h}(2)$ (middle), and $r_{x, h}$ (3) (bottom) with data windowing $(\omega=0.5 \mathrm{rad})$

\begin{tabular}{|c|c|c|c|c|}
\hline & Rectangular & Hamming & Raised cosine & Blackman-Harris \\
\hline \multirow[t]{3}{*}{$N=20$} & 44.681 & 64.099 & 59.064 & 29.857 \\
\hline & 29.430 & 68.188 & 57.975 & 30.152 \\
\hline & 23.188 & 62.440 & 56.895 & 30.953 \\
\hline \multirow[t]{3}{*}{$N=50$} & 34.992 & 78.907 & 116.023 & 147.102 \\
\hline & 31.111 & 74.079 & 113.699 & 146.794 \\
\hline & 30.083 & 72.006 & 108.115 & 146.394 \\
\hline \multirow[t]{3}{*}{$N=100$} & 39.724 & 83.819 & 140.784 & 232.412 \\
\hline & 36.861 & 80.736 & 138.957 & 232.068 \\
\hline & 36.526 & 80.133 & 134.814 & 231.548 \\
\hline \multirow[t]{3}{*}{$N=200$} & 43.934 & 88.024 & 165.346 & 307.083 \\
\hline & 42.565 & 86.604 & 163.824 & 306.904 \\
\hline & 43.468 & 87.454 & 160.715 & 306.121 \\
\hline \multirow[t]{3}{*}{$N=500$} & 50.876 & 94.947 & 199.550 & 320.256 \\
\hline & 53.337 & 97.408 & 198.264 & 327.802 \\
\hline & 60.752 & 104.856 & 196.073 & 320.951 \\
\hline \multirow[t]{3}{*}{$N=1000$} & 94.367 & 138.110 & 236.005 & 323.143 \\
\hline & 62.786 & 106.839 & 235.055 & Not measurable \\
\hline & 58.025 & 102.076 & 234.497 & 327.105 \\
\hline
\end{tabular}


$r_{y, h}(3)$ for a particular sample size and windowing function are within 2-5 dB away from these exception cases. The second category of exceptions occurs when the sample size is too small, e.g., $N=20$ as shown in the first row of Table 3. In this case, the nonlinear factor $w_{k} w_{k+l}$ in (10) prevents the cancellation of the contributions from each period of the sinusoidal signals, which becomes more pronounced the smoother (the lossier in samples) the window is. The respective variance figures for $\left\{r_{y, h}(l), l=1,2,3\right\}$ are around 58 and $30 \mathrm{~dB}$ for raised cosine and $\mathrm{BH}$ windows.

The raised cosine window and $\mathrm{BH}$ window can be considered as successful in reducing the phase dependence. We should note that the results given in Table 3 give information only about the phase dependence mitigation capability of the windows. In order to demonstrate the resulting power loss and the possible trade-offs we perform simulation experiments with these frequency estimators. First, we consider the trade-off between the signal power and the required variance lower bound. In order to demonstrate this, we conduct simulations with a single sinusoid.

\subsection{Trade-off between signal power and variance lower bound}

The processing gain of a data window is given by the ratio of the SNRs before and after data windowing [9]:

$$
\frac{\mathrm{SNR}_{\mathrm{out}}}{\mathrm{SNR}_{\mathrm{in}}}=\frac{1}{N} \frac{\left[\sum_{i=1}^{N} w_{i}\right]^{2}}{\sum_{i=1}^{N} w_{i}^{2}}
$$

which attains its maximum for the case of a rectangular window. While any window, other than rectangular, will cause a drop in SNR, it will also remove some phase dependence. We performed simulations to demonstrate this trade-off between the variance lower bound requirement and the power loss due to data windowing. First, we consider the MSE performance of PISFE as a function of frequency. Then we will consider the variance performance as a function of the SNR. The curves in the later are obtained by averaging the results of 10,000 independent runs and $N=50$.

In Fig. 4 we plot the mean square error (MSE) of the PISFE frequency estimate as a function of angular frequency. Without windowing the MSE of PISFE fluctuates between -35 and $-50 \mathrm{~dB}$, mostly hovering between -40 and $-45 \mathrm{~dB}$. Data windowing removes the frequency dependence and provides MSE gains dependent on the utilized window. The highest gain is obtained with Hamming window usage, followed by raised cosine and $\mathrm{BH}$ window. The smallest MSE values are approximately $-55,-54,-47 \mathrm{~dB}$ for Hamming, raised cosine and $\mathrm{BH}$ windows, respectively, which are obtained at angular frequencies $\pi / 4$ and $3 \pi / 4 \mathrm{rad}$. In Fig. 4 , the curve "c" shows the simulation results of the reformed Pisarenko harmonic decomposition (RPHD) frequency estimator, proposed recently [12], as a frequency estimator for single real sinusoids. This estimator is essentially obtained replacing the AC lags in (12) with some data dependent statistic which can be considered as perturbed AC lag estimates. Its performance is superior to PISFE with non-windowed data and attains bottomline MSE of $-53 \mathrm{~dB}$ at $\pi / 2 \mathrm{rad}$. This result is only about $1 \mathrm{~dB}$

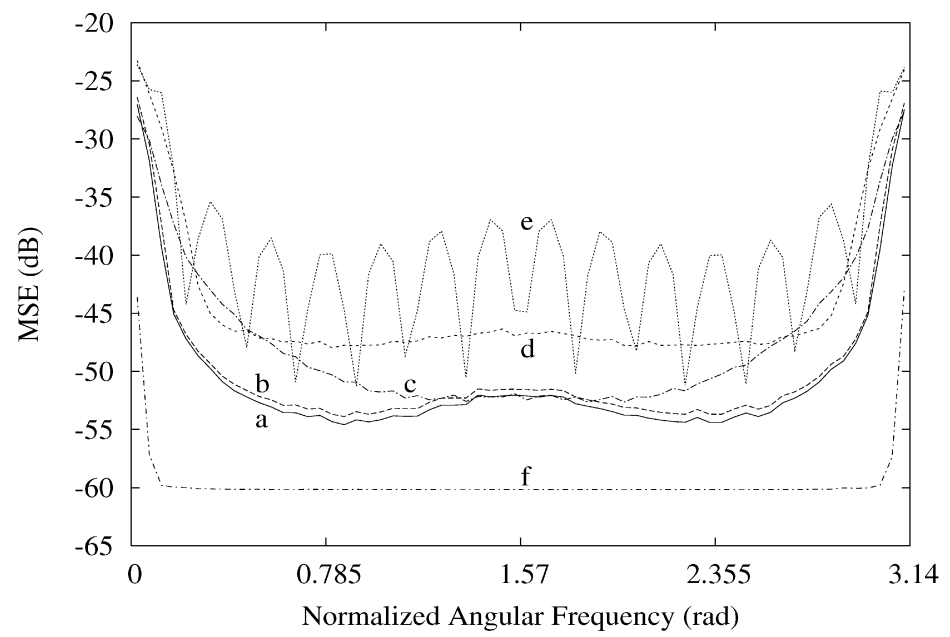

Fig. 4. Variance of the PISFE frequency estimate with various data windows and variance of RPHD versus normalized angular frequency, a: PISFE (Hamming window), b: PISFE (raised cosine window), c: RPHD, d: PISFE (BH window), e: PISFE (no window), f: CRLB (SNR $=20 \mathrm{~dB}, N=50$ ). 


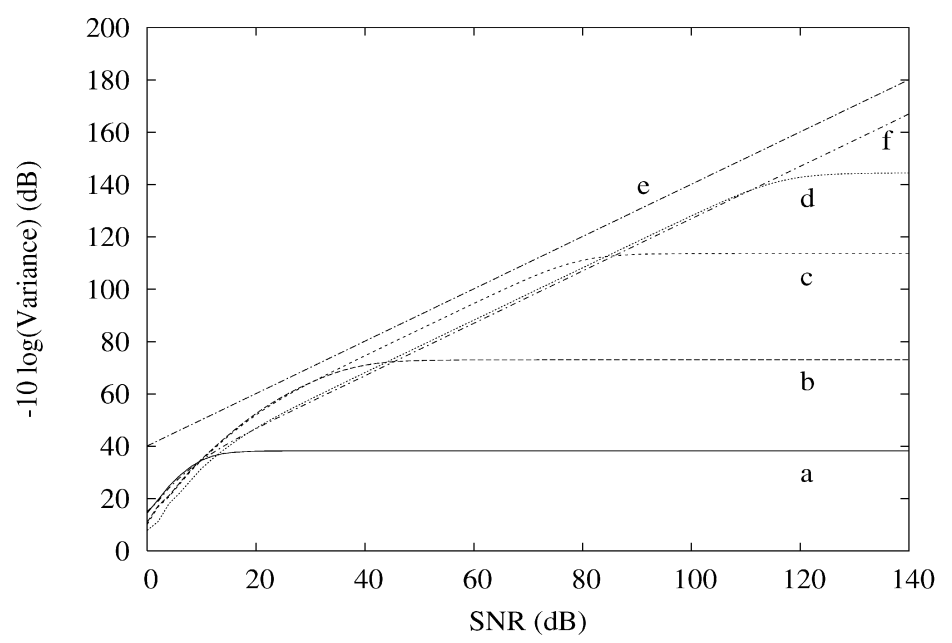

Fig. 5. Variance of the PISFE frequency estimate with various data windows and variance of RPHD versus SNR, a: PISFE (rectangular window, i.e., no window), b: PISFE (Hamming window), c: PISFE (raised cosine window, i.e., Hann window, $\alpha=2$ ), d: PISFE (BH window), e: CRLB, f: $\operatorname{RPHD}(\omega=0.5 \mathrm{rad}, N=50)$.

smaller than the MSE of PISFE with raised cosine window and just equal to the MSE of PISFE with Hamming window. At other frequencies, the latter two estimators are superior to the RPHD. While PISFE with BH window depicts a performance with the least dependence on frequency, it causes at the same time the highest power loss among all of the PISFE variants with data windowing. The plotted curves are the averages of 1000 independent runs at $N=50$. We should also note that the MSE of PISFE obtained via numerical integration using the derived analytical pdf expression closely matches the simulation results in the angular frequency $\omega$ interval of $[0.16,2.98] \mathrm{rad}$.

In Fig. 5, the variance of PISFE is plotted against SNR for $\omega=0.5 \mathrm{rad}$. At low SNR values, the phase dependence is eclipsed and a performance loss of $1.5 \mathrm{~dB}$ in the variance figures are observed due to the power loss caused by data windowing for SNR $<10 \mathrm{~dB}$. As the SNR improves, the rectangular window case shown by curve "a" fails to follow the linear trend of the Cramer-Rao lower bound (CRLB). Instead it saturates quickly at around SNR $=15 \mathrm{~dB}$, and the flattening at a level of $-40 \mathrm{~dB}$, is the outcome of the phase dependence limitation. On the other hand, the curves of windowed cases "b," "c," and "d," show the improved elimination of the phase dependence. In fact, the saturation points reveal themselves for the Hamming, raised cosine and BH windows, respectively, at 30, 75, and 115 $\mathrm{dB}$ SNR values. Similarly the noise floors of these windowed estimates gets realized at $-70,-110$, and $-150 \mathrm{~dB}$, respectively. The performance in the case of raised cosine window is $6 \mathrm{~dB}$ inferior to the CRLB and the compromise for an additional improvement in phase dependence offered by BH window is the loss of signal power of about another $6 \mathrm{~dB}$. The performance of RPHD, as shown in curve " $\mathrm{f}$ " is similar to the non-windowed PISFE estimator for $\mathrm{SNR}<10 \mathrm{~dB}$, but in contrast, it follows the trend of the CRLB curve as SNR increases. However, it can never approach the CRLB curve by less than $12 \mathrm{~dB}$. This RPHD performance is worse than the BH-windowed PISFE one. These simulation results are valid for any $\omega$ with possible singular point exceptions for PISFE without windowing $[4,13]$ as can be seen in Fig. 4. One should notice that the variance lower bounds attained by windows are strongly correlated with the variances of the phase-dependent AC lags as given in Table 3. At these high SNR values, AWGN becomes negligible when compared to the phase-dependent indeterminacy.

As for Fig. 5, a qualitative comparison of the estimators can be made by pointing out the trade-off between the power loss and the SNR operation range. Here we focus on the SNR operation range beyond the value at which the estimator variance curves cross each other. For example, at SNR $=10 \mathrm{~dB}$ the non-windowed PISFE starts to saturate and it has the same variance as PISFE with Hamming window. So, the higher SNR operation limit of PISFE is pushed from 10 to 31, 84, $110 \mathrm{~dB}$ and noise-free cases when PISFE with Hamming, raised cosine and $\mathrm{BH}$ windows and RPHD, respectively, are used. The corresponding incremental power losses taking non-windowed PISFE as the starting reference, are 1.3, 0.5, 6 , and $1.3 \mathrm{~dB}$, respectively. According to this comparison PISFE with raised cosine window seems to be the best compromise since it extends the SNR operation range to a satisfactory level of $84 \mathrm{~dB}$ with a total cost of $1.8 \mathrm{~dB}$ power loss. 


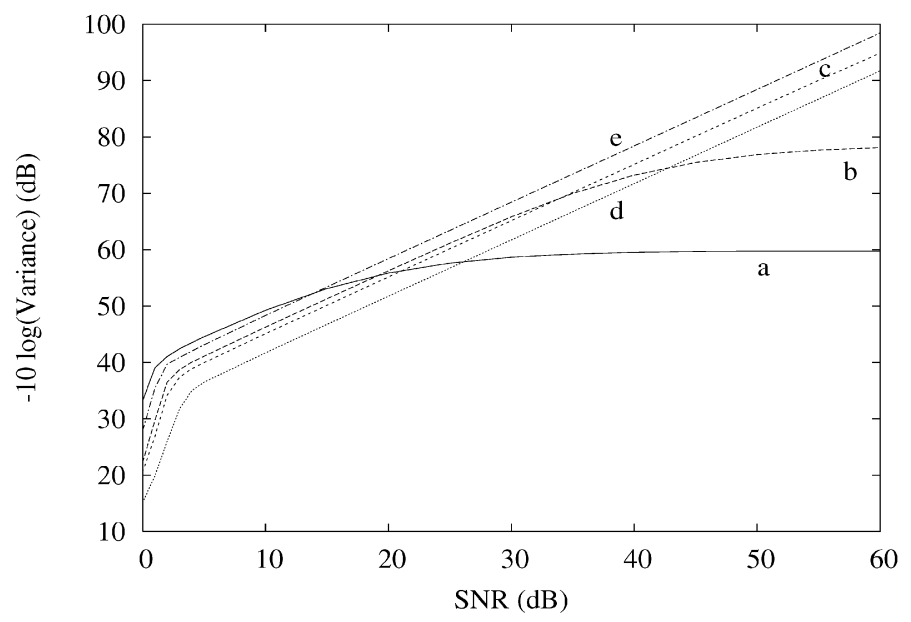

Fig. 6. Variance of PC-AR frequency estimate with various data windows versus SNR averaged on the frequency axis, a: rectangular window, b: Hamming window, c: raised cosine window, d: BH window, e: estimator based on non-Toeplitz AC matrix estimates with rectangular window $(N=50, M=20)$.

In Fig. 6, we show similar considerations for the PC-AR frequency estimator. The curves reflect averaging over different frequencies. The AC matrix is $20 \times 20$. In contrast to Fig. 5, we remark the following: (i) The loss of signal power is much more evident, as one compares curve "a" vis-a-vis curves $b, c, d$ in the $0-20 \mathrm{~dB}$ SNR range in both Figs. 5 and 6; (ii) The PC-AR method with the Hamming window catches up with the Tufts-Kumaresan (TK) frequency estimator [14] based on the non-Toeplitz AC matrix. The performance difference is only $2.5 \mathrm{~dB}$ at the variance level of $-60 \mathrm{~dB}$. Due to the relatively low variance lower bound with Hamming window, the raised cosine window is more preferable albeit at an additional power loss of $1 \mathrm{~dB}$.

\subsection{Resolving capability in the case of two close sinusoids}

In the case of multiple sinusoids, the analysis for the single sinusoidal case is exactly applicable as long as these sinusoids are well resolved, that is the minimum frequency difference is significantly greater than the Fourier resolution limit of $2 \pi / N$. If this is not the case, then one should consider the price paid for the phase dependence reduction, that is, the loss of resolution. We set out to choose a window with smaller power loss in order to limit the loss of resolution. But we found out that a choice based solely on the trade-off between the power loss and the variance lower bound was satisfactory. Our experiments showed that raised cosine window leads to better resolution performance with respect to both Hamming window and $\mathrm{BH}$ window when the frequencies were at the half the Fourier resolution limit. Therefore we present in the sequel the results with raised cosine window.

To understand the effect of phase dependence on the resolution, we start considering the histograms of estimated frequencies, when the sinusoidal frequencies differ by $2 \pi / N(\mathrm{SNR}=40 \mathrm{~dB})$. The location of the true frequencies are shown by vertical lines in these figures. In the first two of them, Figs. 7A and 7B, we also see the histograms of the estimated frequencies under raised-cosine and rectangular windows, respectively. The histograms clearly show that without data windowing, the sinusoids are not resolved in every realization whereas they are resolved perfectly when a data window is used. As a measure of resolution capability we adopt the resolution probability, where it is required that the inequality

$$
\left(\hat{\omega}_{1}-\omega_{1}\right)^{2}+\left(\hat{\omega}_{2}-\omega_{2}\right)^{2}<\min \left(\left(\hat{\omega}_{1}-\omega_{\text {mid }}\right)^{2}+\left(\hat{\omega}_{2}-\omega_{\text {mid }}\right)^{2}, 2\left(\omega_{2}-\omega_{1}\right)^{2}\right)
$$

be satisfied for resolved frequencies, and where $\left\{\hat{\omega}_{i}, i=1,2\right\}$ and $\omega_{\text {mid }}$ correspond to the estimate and the arithmetic average of the sinusoidal frequencies $\left\{\omega_{i}, i=1,2\right\}$, respectively, and $\min (\cdot, \cdot)$ denotes the minimum of its arguments. The first argument of the $\min (\cdot, \cdot)$ function assures that the estimated peaks are not both located in the vicinity of $\omega_{\text {mid }}$ whereas the second argument serves to reject the outliers in the histograms as in Fig. 7B. This criterion successfully classifies the frequency estimates into resolved and unresolved sets as shown by the histograms in Fig. 7C. Note that, the histograms of the frequency estimates in Fig. 7A have a smeared form of the pdf of a phase- 


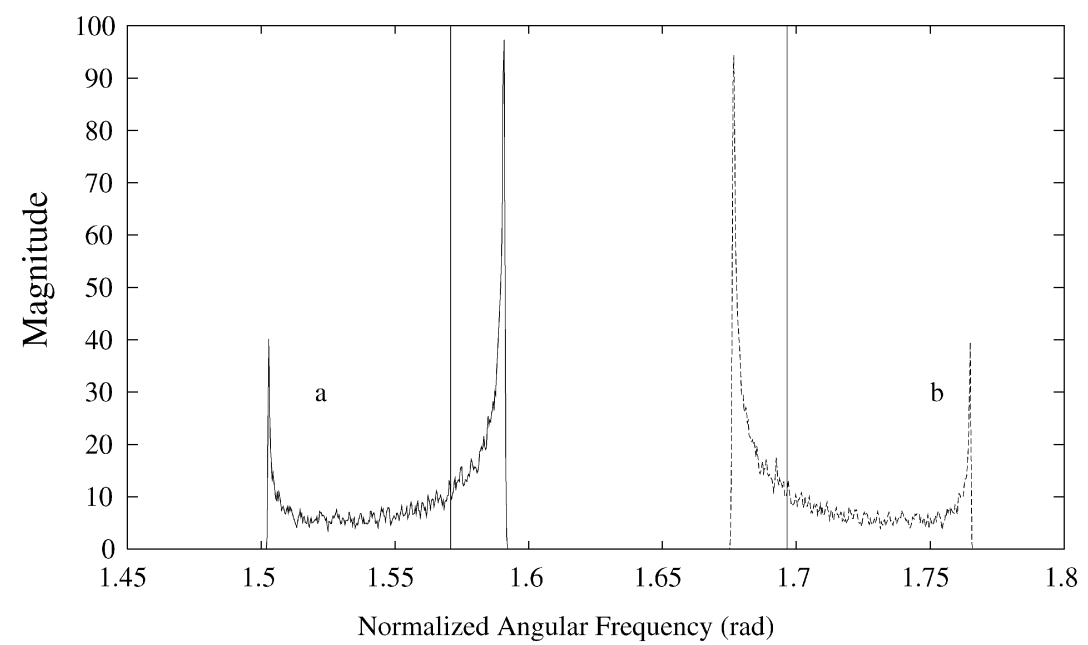

(A) The two frequency estimates (raised cosine data window)

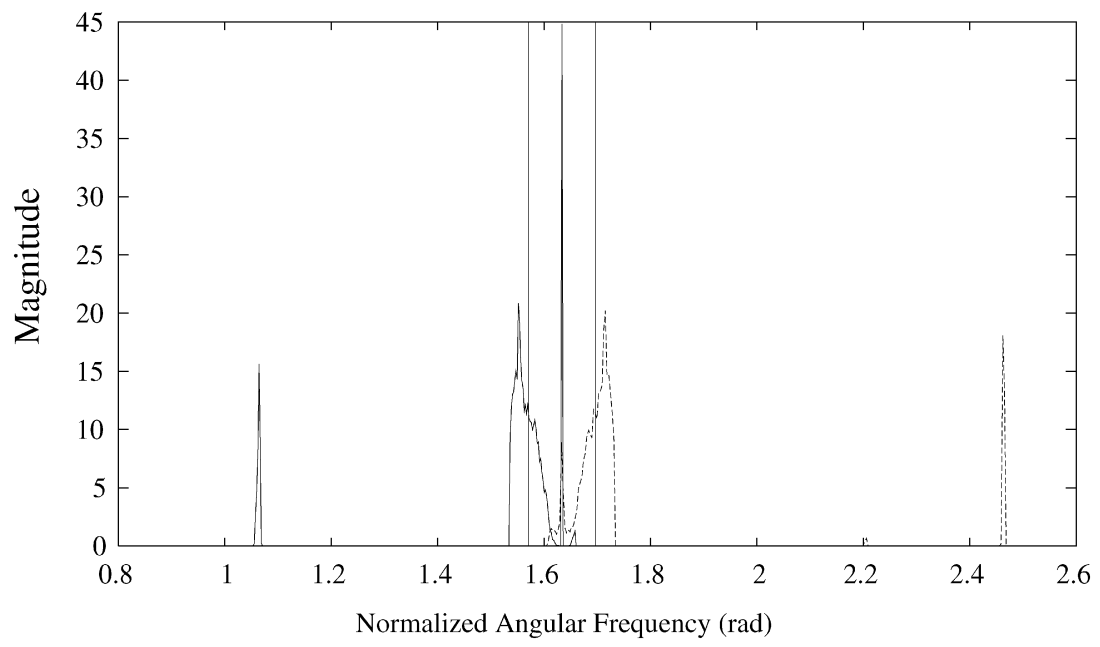

(B) The two frequency estimates (no data window)

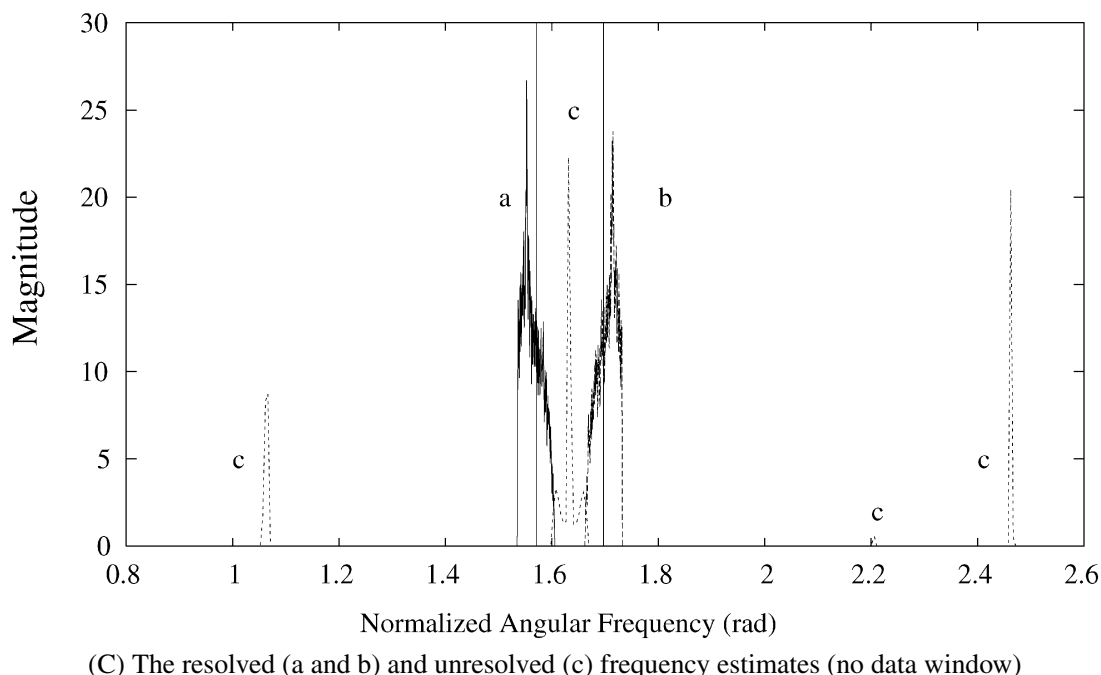

Fig. 7. Histograms of the MUSIC frequency estimates $\left(\omega_{1}=1.5708 \mathrm{rad}, \omega_{2}=1.6965 \mathrm{rad}, \omega_{2}-\omega_{1}=2 \pi / N \mathrm{rad}, N=50, M=20,100,000\right.$ noise realizations). 
noise-dependent component. Such a pdf was derived for the single sinusoidal case and it was shown in Fig. 2 with curve "d."

In Figs. 8A, 8B, and 8C, we plot the resolution probabilities of MUSIC frequency estimator with and without data windowing against SNR, for two sinusoids where their frequency difference is $4 \pi / N, 2 \pi / N$, and $\pi / N \mathrm{rad}$, respectively, going from a well resolved case toward an underresolved case of half of the Fourier resolution limit. In these figures we plot the resolution probabilities of the non-windowed, windowed, and non-Toeplitz AC matrixbased variants of the MUSIC frequency estimator. The data window is the raised cosine window which achieves moderate phase dependence reduction at a small expense in the estimation variance. When the sinusoids are well resolved, the phase-dependent variance is ineffective on the resolution of the frequency estimator and the use of a data window only decreases the resolution probability of the frequency estimator. At a resolution probability of 0.9, MUSIC frequency estimator based on the non-Toeplitz AC matrix estimate and the one utilizing the raised cosine window require approximately 1.6 and $2.4 \mathrm{~dB}$ more signal power as compared to no data windowing. The slightly inferior performance of the non-Toeplitz AC matrix based estimator with respect to the Toeplitz AC matrix based estimator in Fig. 8A, is in accordance with the behavior of the PC-AR counterparts of these estimators in Fig. 6 for $\mathrm{SNR}<13 \mathrm{~dB}$.

When the frequency difference equals $2 \pi / N$, the gain due to phase dependence suppression becomes more effective than loss due the spectral broadening. This happens at SNRs higher than $5 \mathrm{~dB}$ and the windowed MUSIC frequency estimator attains unity resolution probability at SNR as low as $10 \mathrm{~dB}$ whereas the non-windowed estimator can only achieve asymptotically a resolution probability of 0.78 . Despite the spectral broadening caused by the data window the resolution performance of the non-Toeplitz AC matrix-based estimator is about $2.5 \mathrm{~dB}$ inferior to that of the windowed estimator for resolution probabilities greater that 0.5 . This is due to the fact that the inferiority of the non-Toeplitz AC matrix-based frequency estimators with respect to their Toeplitz AC matrix based counterparts at low SNRs increases as the frequency difference of the sinusoids decreases and this effect is higher when compared to the resolution loss due to data windowing. Fig. $8 \mathrm{C}$ depicts that when the frequency difference of the sinusoids is half of the Fourier resolution limit, the windowed MUSIC frequency estimator can no longer attain unity resolution probability and saturates at a level of 0.87 whereas the non-Toeplitz AC matrix based frequency estimator achieves this at $\mathrm{SNR}=35 \mathrm{~dB}$. The asymptotic resolution probability for the non-windowed Toeplitz AC matrix based frequency estimator is as low as 0.58 , in this case.

\section{Discussion and conclusions}

In this study we considered the phase dependence problem specific to the sinusoidal frequency estimation using Toeplitz AC matrix estimates and proposed data windowing as a tool for mitigation of this effect. Lag windowing (quadratic tapering [15]) is out of question for the phase dependence suppression problem. The only similarity between the effects of data windowing (linear tapering [15]) and lag windowing on the AC lags is in the fact that the means of the AC lags are multiplied by a constant factor due to data windowing, as given in (B.2). This could be also accomplished by a corresponding lag window where the lag window coefficient for that particular AC lag is chosen as equal to the factor of that $\mathrm{AC}$ lag in (B.2). However, when you multiply a random variable with a constant, what you would expect will be just a corresponding scaling of the pdf of that random variable. This means that other than a change in the support and magnitudes, the pdf will maintain its original form, particularly the twin-peaked form at high SNR values in our case.

We should mention that data windowing would be also useful for the signal models with deterministic but unknown phases. In those cases, the pdf of the phase-dependent AC lag component becomes a unit impulse which causes an unknown deterministic shift when convolved with the Gaussian component of the pdf. The proposed method will also decrease the amount of this bias in the AC lags.

The main conclusions of the paper can be listed as follows:

- The Toeplitz AC matrix estimates can be analyzed as a sum of two independent variates, a phase-dominated term and a noise-dominated term. The pdf of the lag coefficient, computed as the convolution of the pdfs of the two random variates, matches very well the experimentally determined histogram.

- The phase dependence phenomenon proves to be detrimental in small sample size and high SNR applications, i.e., at least above $20 \mathrm{~dB}$. For example for a very clean sinusoid at $\mathrm{SNR}=40 \mathrm{~dB}$, data windowing decreases the 


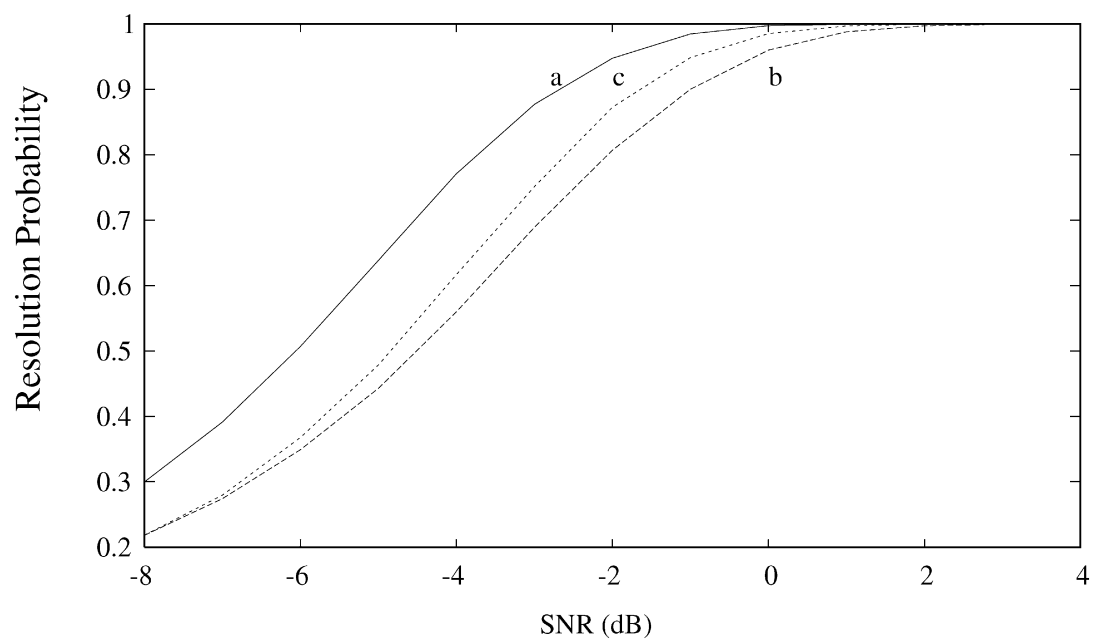

(A) $\omega_{2}-\omega_{1}=4 \pi / N \mathrm{rad}$ (twice DFT resolution limit)

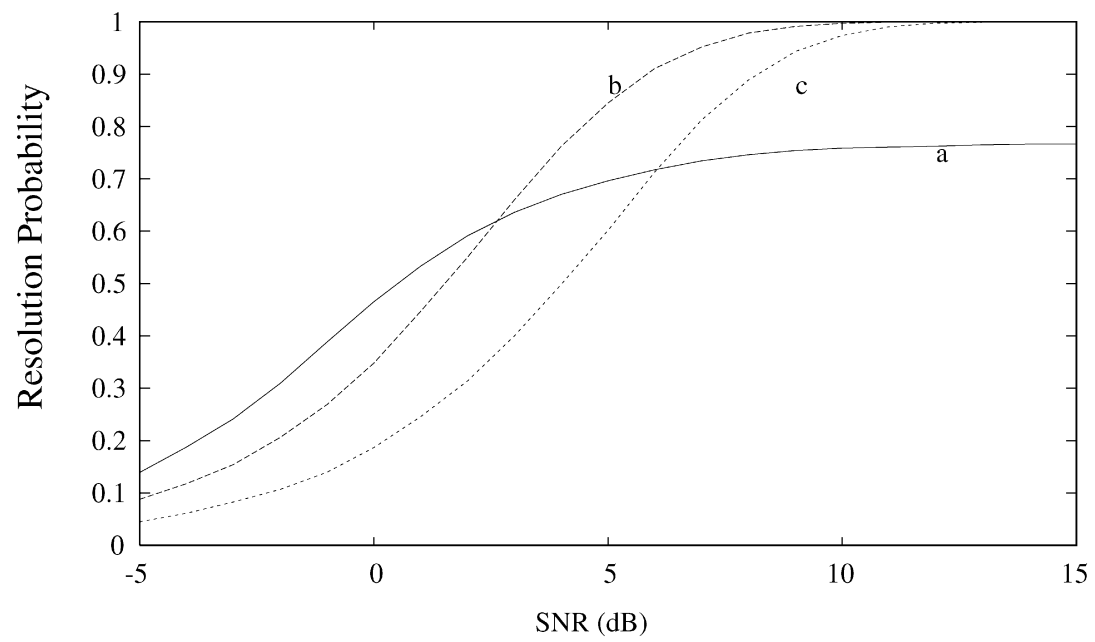

(B) $\omega_{2}-\omega_{1}=2 \pi / N$ rad (DFT resolution limit)

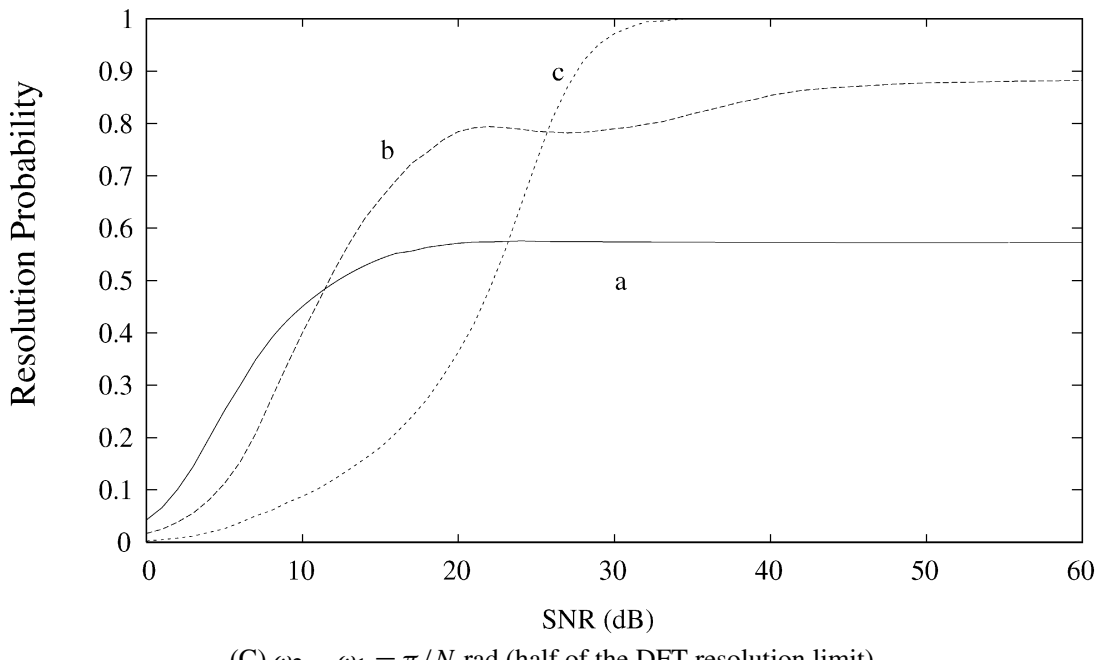

(C) $\omega_{2}-\omega_{1}=\pi / N$ rad (half of the DFT resolution limit)

Fig. 8. Resolution probability of the MUSIC frequency estimators versus SNR, a: rectangular window (Toeplitz AC matrix), b: raised cosine window (Toeplitz AC matrix), c: rectangular window (non-Toeplitz AC matrix) $\left(\omega_{1}=1.57 \mathrm{rad}, N=50, M=20\right)$. 
estimated frequency variance by a factor of $300(25 \mathrm{~dB})$. On the other hand windowing is not useful for SNRs lower than $20 \mathrm{~dB}$.

- In summary, the performance of frequency estimators based on Toeplitz AC methods improves with data windowing approaching that of non-Toeplitz AC methods, albeit with some loss in signal power. However, since the beneficial operational region of windowing is in the high SNR range, this loss in power is more than compensated by the suppression of phase dependence. Recall again that the main motivation to save and promote Toeplitz ACbased estimators to the level of non-Toeplitz varieties was their computational advantage. Windowing achieves this with a small loss in resolution power.

\section{Appendix A. Derivation of the pdf of $r_{x, h}(l)$}

We rewrite $r_{x, h}(l)$ from (6) as

$$
\begin{aligned}
r_{x, h}(l) & =\frac{1}{N-l}\left(\cos (2 \phi)[\underbrace{\left[\sum_{k=1}^{N-l} \cos (\omega(2 k+l))\right]}_{C(N, l)}-\sin (2 \phi) \underbrace{\left[\sum_{k=1}^{N-l} \sin (\omega(2 k+l))\right]}_{S(N, l)})\right. \\
& =\underbrace{\frac{1}{N-l} \sqrt{C^{2}(N, l)+S^{2}(N, l)}}_{d_{x}(l)} \cos \underbrace{\left(2 \phi-\tan ^{-1}\left(\frac{S(n, l)}{C(n, l)}\right)\right)}_{\zeta} .
\end{aligned}
$$

The argument of cosine function in (A.2), $\zeta$, is uniformly distributed in $(0,2 \pi)$. Let us define $\gamma=\cos \zeta$. One can obtain the pdf of $\gamma$ according to the rules of transforming random variables as

$$
f_{\Gamma}(\gamma)=\frac{1}{\pi \sqrt{1-\gamma^{2}}}, \quad-1 \leqslant \gamma \leqslant 1 .
$$

Since the multiplier of $\gamma$ in (A.2) is a constant, one can easily obtain the pdf given in (7) through another variable transformation.

\section{Appendix B. PDF of the AC-lags after data windowing for the single sinusoidal case}

Let $y_{k}=w_{k} x_{k}$ where $\left\{w_{k}, k=1,2, \ldots, N\right\}$ represents a data window [9,11]. For a rectangular window function the asymptotic pdf of

$$
r_{y}(i)=r_{x}(i)=r_{x, h}(i)+r_{x, g}(i)
$$

is the convolution of the pdf's of $r_{x, h}(i)$ and $r_{x, g}(i)$ referred to as $f_{R_{x, h}}^{(i)}\left(r_{x, h}\right)$ and $f_{R_{x, g}}^{(i)}\left(r_{x, g}\right)$, respectively, under the independence assumption. $f_{R_{x, h}}^{(i)}\left(r_{x, h}\right)$ has the form in (7) and $f_{R_{x, g}}^{(i)}\left(r_{x, g}\right)$ was found to be Gaussian with mean and variance given by (4) and (5), respectively [8]. It can be shown easily that

$$
f_{R_{y}}^{(i)}\left(r_{y}\right)=f_{R_{y, h}}^{(i)}\left(r_{y, h}\right) * f_{R_{y, g}}^{(i)}\left(r_{y, g}\right),
$$

where

$$
r_{y}(i)=r_{y, h}(i)+r_{y, g}(i) .
$$

In the case of a general data window $f_{R_{y, h}}^{(i)}\left(r_{y, h}\right)$ again has the form of (7), but now $d_{y}(i)$ is given by

$$
d_{y}(i)=\frac{1}{N-i} \sqrt{\left(\sum_{k=1}^{N-i} w_{k} w_{k+i} \cos (\omega(2 k+i))\right)^{2}+\left(\sum_{k=1}^{N-i} w_{k} w_{k+i} \sin (\omega(2 k+i))\right)^{2}} .
$$

The distribution of $r_{y, g}(i)$ 's are again Gaussian due to the CLT with the first and second moments scaled according to the windowing function. These moments can be derived based on the derivation in [8] for the non-windowed data. 
The mean of the $r_{y}(i)$ is

$$
\mu_{y, g}(i)=E\left\{\frac{1}{N-i} \sum_{k=1}^{N-i} w_{k} x_{k} w_{k+i} x_{k+i}\right\}=\frac{1}{N-i} \sum_{k=1}^{N-i} w_{k} w_{k+i} \mu_{x, g}(i),
$$

where $\mu_{x, g}(i)=E\left\{r_{x, g}(i)\right\}=\cos (\omega i)+\sigma_{n}^{2} \delta_{i, j}$ with

$$
\delta_{i, j}= \begin{cases}1 & \text { for } i=j, \\ 0 & \text { for } i \neq j .\end{cases}
$$

The covariance of $r_{y, g}(i)$ and $r_{y, g}(j)$ can be calculated as

$$
\sigma_{y, g}^{2}(i, j) \equiv \operatorname{cov}\left(r_{y, g}(i), r_{y, g}(j)\right)=E\left\{\left(r_{y, g}(i)-E\left(r_{y, g}(i)\right)\right)\left(r_{y, g}(j)-E\left(r_{y, g}(j)\right)\right)\right\} .
$$

Inserting (B.2) into (B.3) and expanding it one obtains

$$
\begin{aligned}
\sigma_{y, g}^{2}(i, j)= & \frac{1}{(N-i)(N-j)} \sum_{k=1}^{N-i} \sum_{l=1}^{N-j} w_{k} w_{k+i} w_{l} w_{l+i} \\
& \times E\left\{\left(\left(s_{k}+n_{k}\right)\left(s_{k+i}+n_{k+i}\right)-\mu_{x, g}(i)\right)\left(\left(s_{l}+n_{l}\right)\left(s_{l+i}+n_{l+i}\right)-\mu_{x, g}(j)\right)\right\},
\end{aligned}
$$

where $\left\{s_{k}\right\}$ is as in (1). Since the sequences $\left\{s_{k}\right\}$ and $\left\{n_{k}\right\}$ are independent the fourth order terms in the expectation are calculated mainly as multiplications of second order terms, e.g.,

$$
E\left\{s_{k} s_{k+i} n_{l} n_{l+j}\right\}=E\left\{s_{k} s_{k+i}\right\} E\left\{n_{l} n_{l+j}\right\}=\mu_{s}(i) \sigma_{n}^{2} \delta_{l, l+j} .
$$

One of the exceptions is the term $E\left\{s_{k} s_{k+i} s_{l} s_{l+j}\right\}$. Considering its contribution to the summation in (B.4) we may replace this term by

$$
E\left\{s_{k} s_{k+i}\right\} E\left\{s_{l} s_{l+j}\right\}=\cos (\omega i) \cos (\omega j) .
$$

The other exception is the term $E\left\{n_{k} n_{k+i} n_{l} n_{l+j}\right\}$ which is given as [16]

$$
E\left\{n_{k} n_{k+i} n_{l} n_{l+j}\right\}=\sigma_{n}^{4}\left\{\delta_{k, l} \delta_{k+i, l+j}+\delta_{k, k+i} \delta_{l, l+j}+\delta_{k, l+j} \delta_{l, l+k}\right\} .
$$

After tedious steps one obtains

$$
\begin{aligned}
\sigma_{y, g}^{2}(i, j)= & \frac{\sigma_{n}^{2}}{(N-i)(N-j)} \sum_{k=1}^{N-i} \sum_{l=1}^{N-j}\left(w_{k} w_{k+i} w_{l} w_{l+i}\right)\left\{\mu_{s}(k-l) \delta_{k+i, l+j}+\mu_{s}(k-(l+j)) \delta_{k+i, l}\right. \\
& +\mu_{s}((k+i)-l) \delta_{k, l+j}+\mu_{s}((k+i)-(l+j)) \delta_{k+i, l+j} \\
& \left.+\sigma_{n}^{2}\left[\delta_{k, l} \delta_{k+i, l+j}+\delta_{k, k+i} \delta_{l, l+j} \delta_{k, l+j} \delta_{l, k+i}+\delta_{i, 0} \delta_{j, 0}\right]\right\} .
\end{aligned}
$$

When $i=j$, (B.5) simplifies to

$$
\sigma_{y, g}^{2}(i, i)=\sigma_{y, g}^{2}(i)=\frac{\sigma_{n}^{2}}{(N-i)^{2}}\left\{\sum_{k=1}^{N-i} 2 w_{k}^{2} w_{k+i}^{2}+\sum_{k=1}^{N-2 i} 2 \cos (2 i \omega) w_{k} w_{k+i}^{2} w_{k+2 i}+\sigma_{n}^{2} \sum_{k=1}^{N-i} w_{k}^{2} w_{k+i}^{2}\right\} .
$$

For the rectangular window the asymptotic value of (B.6)

$$
\lim _{N \rightarrow \infty} \sigma_{y, g}^{2}(i)=\frac{\sigma^{2}}{N}\left[2+2 \cos (2 i \omega)+\sigma^{2}\right]=\frac{\sigma^{4}}{N}\left(1+4 \eta \cos (i \omega)^{2}\right)
$$

with $\eta=1 / \sigma_{n}^{2}$, attains the form given in [8]. In the derivation of the pdf of PISFE the covariances $\sigma_{y, g}^{2}(1), \sigma_{y, g}^{2}(2)$, and $\sigma_{y, g}^{2}(1,2)=\sigma_{y, g}^{2}(2,1)$ are used. The first two of these covariances are obtained using (B.6). The others are found using (B.5) as

$$
\begin{aligned}
\sigma_{y, g}^{2}(1,2)= & \frac{\sigma_{n}^{2}}{(N-1)(N-2)}\left\{\sum_{k=1}^{N-2} \cos (\omega)\left[w_{k} w_{k+1} w_{k+2}\left(w_{k}+w_{k+2}\right)\right]\right. \\
& \left.+\sum_{k=1}^{N-3} \cos (3 \omega)\left[w_{k} w_{k+3}\left(w_{k+1}^{2}+w_{k+2}^{2}\right)\right]\right\} .
\end{aligned}
$$


Although these covariance expressions correspond to asymptotic values, they are observed to work well for short data records of, e.g., $N=50$. Finally, the windowing causes a bias in the AC lag estimates as seen in (B.2). This bias will be removed by replacing $r_{y}(i)$ with

$$
\frac{(N-i) r_{y}(i)}{\sum_{k=1}^{N-i} w_{k} w_{k+i}}
$$

\section{Appendix C. Derivation of PISFE PDF}

To derive the pdf of PISFE, $f$ ( $\hat{\omega}_{\text {PISFE }}$ ), we start with finding the pdf $f(\psi)$. This pdf can be obtained by using the statistics of $r_{y, g}(1)$ and $r_{y, g}(2)$ assuming the effect of the phase dependence is made negligible with data windowing. AC-lag estimates are approximately jointly Gaussian and their means and covariances are found in Appendix B. For the ease of the notation we let $r_{y, g}(1)=r_{1}$ and $r_{y, g}(2)=r_{2}$. Using the variable transformation

$$
z_{1}=r_{1}, \quad z_{2}=\psi
$$

the Jacobian of the transformation $J\left(z_{1}, z_{2}\right)$ is found as

$$
J\left(z_{1}, z_{2}\right)=\frac{1}{4 z_{1}}\left[1+\operatorname{sgn}\left(z_{1}\right) A_{1}\left(z_{2}\right)\right]
$$

where

$$
\operatorname{sgn}\left(z_{1}\right)= \begin{cases}1 & \text { for } z_{1}>0 \\ 0 & \text { for } z_{1}=0 \\ -1 & \text { for } z_{1}<0\end{cases}
$$

So one obtains the joint density of $z_{1}$ and $z_{2}$

$$
f_{Z_{1}, Z_{2}}\left(z_{1}, z_{2}\right)=\frac{f_{R_{1}, R_{2}}\left(z_{1}, z_{1} A_{0}\left(z_{2}\right)\right)}{\left|\frac{1}{4 z_{1}}\left(1+\operatorname{sgn}\left(z_{1}\right) A_{1}\left(z_{2}\right)\right)\right|}
$$

and the corresponding marginal density of $\psi$ as

$$
f_{\Psi}(\psi)=\int_{-\infty}^{\infty} \frac{f_{R_{1}, R_{2}}\left(z_{1}, z_{1} A_{0}(\psi)\right)}{\left|\frac{1}{4 z_{1}}\left(1+\operatorname{sgn}\left(z_{1}\right) A_{1}(\psi)\right)\right|} d z_{1}
$$

This equation can be expressed as

$$
f_{\Psi}(\psi)=K_{1} \int_{-\infty}^{\infty} \exp \left\{-\frac{1}{2}\left[A_{2}(\psi) z_{1}^{2}+A_{3}(\psi) z_{1}+A_{4}\right]\right\} d z_{1},
$$

which can be solved using integral tables [17, p. 302] resulting in the expression of (14).

\section{References}

[1] S.M. Kay, Modern Spectral Estimation: Theory and Application, Prentice Hall, New Jersey, 1988.

[2] W.H. Press, B.P. Flannery, S.A. Teukolsky, W.T. Vetterling, Numerical Recipes in C: The Art of Scientific Computing, Cambridge Univ. Press, Cambridge, 1992.

[3] W.F. Trench, Numerical solution of the eigenvalue problem for efficiently structured Hermitian matrices, Linear Algebra Appl. 154-156 (1991) 415-432.

[4] K.W. Chan, H.C. So, An exact analysis of Pisarenko's single-tone frequency estimation algorithm, Signal Process. 83 (3) (2003) 685-690.

[5] H.C. So, A comparative study of three recursive least-squares algorithms for single tone frequency tracking, Signal Process. 83 (9) (2003) 2059-2062.

[6] P. Stoica, R. Moses, Introduction to Spectral Analysis, Prentice Hall, New Jersey, 1997.

[7] S.M. Kay, Robust detection by autoregressive spectrum analysis, IEEE Trans. Acoust. Speech Signal Process. ASSP-30 (1982) $256-269$.

[8] P. Stoica, T. Söderström, F.-N. Ti, Overdetermined Yule-Walker estimation of the frequencies of multiple sinusoids: Accuracy aspects, Signal Process. 16 (1989) 155-174.

[9] F.J. Harris, On the use of windows for harmonic analysis with the discrete Fourier transform, Proc. IEEE 66 (1) (1978) 53-83. 
[10] Y.F. Pisarenko, The retrieval of harmonics from a covariance function, Geophys. J. R. Astron. Soc. 33 (1973) $347-366$.

[11] A.H. Nuttall, Some windows with very good sidelobe behavior, IEEE Trans. Acoust. Speech Signal Process. ASSP-29 (1) (1981) 84-91.

[12] H.C. So, K.W. Chan, Reformulation of Pisarenko harmonic decomposition method for single-tone frequency estimation, IEEE Trans. Signal Process. 52 (4) (2004) 1128-1135.

[13] H. Sakai, Statistical analysis of Pisarenko's method for sinusoidal frequency estimation, IEEE Trans. Acoust. Speech Signal Process. ASSP32 (1) (1984) 95-101.

[14] D.W. Tufts, R. Kumaresan, Estimation of frequencies of multiple sinusoids: Making linear prediction perform like maximum likelihood, Proc. IEEE 70 (9) (1982) 975-989.

[15] C. Van Schooneveld, D.J. Frijling, Spectral analysis: On the usefulness of linear tapering for leakage suppression, IEEE Trans. Acoust. Speech Signal Process. ASSP-29 (2) (1981) 323-329.

[16] A. Papoulis, Probability, Random Variables and Stochastic Processes, third ed., McGraw-Hill, New York, 1991.

[17] M.A. Abramowitz, I.A. Stegun, Handbook of Mathematical Functions, Dover, New York, 1965.

Mustafa A. Altınkaya was born in Rize, Turkey, in 1965. He received the B.S., M.S., and Ph.D. degrees in electrical and electronics engineering from Boğaziçi University, Istanbul, Turkey, in 1987, 1990, and 1996, respectively. From 1989 to 1990 he was a research associate in TUBITAK Marmara Research Center, Kocaeli, Turkey. From 1991 to 1996 he was a research and teaching assistant in Boğaziçi University. He worked as a part-time instructor in Air Force Academy, Naval Academy and Boğaziçi University during periods from 1995 to 1998. In 1998 he joined the Electrical and Electronics Engineering Department at Izmir Institute of Technology where he has been an Assistant Professor. He is the author of more than 20 publications. His research interests are mainly in statistical signal processing and its applications in telecommunications, structural dynamic systems, and bioinformatics.

Dr. Emin Anarım received the B.Sc. (First Class, Honours), M.Sc., and Ph.D. degrees in electronic and electrical engineering from Boğaziçi University, Istanbul, Turkey, in 1981, 1983, and 1985, respectively. After spending 17 years in Turkish Air Force Army as an officer, in January 1992 he joined the Department of Electrical and Electronics Engineering of Boğaziçi University, Istanbul, Turkey, where he is presently a full-time Professor. His current research interests are in the field of multimedia wireless communication, image and video sequence coding, low-level image processing algorithms, software quality measurements, target identification, tracking, data compression algorithms, information and network security. More than 120 papers carrying his name have appeared in various journals and conference proceedings. Dr. Emin Anarim is also presently an adjunct Professor of George Washington University. He has given several courses to industry and government institutions on signal processing, video coding, secure telecommunications, mobile communications, lawful interception techniques in telecommunications, crytographic techniques, and network security.

Bülent Sankur received the B.S. degree in electrical engineering from Robert College, Istanbul, and completed the M.Sc. and Ph.D. degrees at Rensselaer Polytechnic Institute, New York, USA. He has been teaching at Boğaziçi (Bosphorus) University in the Department of Electrical and Electronic Engineering. His research interests are in the areas of digital signal processing, image and video compression, biometry, cognition, and multimedia systems. Dr. Sankur has held visiting positions at University of Ottawa, Technical University of Delft, and Ecole Nationale Supérieure des Télécommunications, Paris. He was the chairman of ICT '96: International Conference on Telecommunications and of EUSIPCO '05: The European Conference on Signal Processing as well as technical chairman of ICASSP ' 00 . 